\title{
Inhibition of the ubiquitin-proteasome system sensitizes TRAIL- resistant prostate cancer cells by up-regulation of death receptor 5
}

\author{
YOU-JIN LEE, JAE-WON SEOL, JAE-KYO JEONG, MYUNG-HEE MOON and SANG-YOUEL PARK \\ Center for Healthcare Technology Development, Bio-Safety Research Institute, College of Veterinary Medicine, \\ Chonbuk National University, Jeonju, Jeonbuk 561-756, Republic of Korea
}

Received June 21, 2011; Accepted August 10, 2011

DOI: $10.3892 / \mathrm{mmr} .2011 .558$

\begin{abstract}
Tumor necrosis factor (TNF)-related apoptosisinducing ligand (TRAIL) is a type II transmembrane cytokine and a potent inducer of apoptosis in cancer cells. However, some cancer cells, particularly prostate cancer cells, such as LNCaP cells, were found to be resistant to TRAIL. In the present study, we demonstrate that the proteasome inhibitor ALLN significantly enhanced TRAIL-induced apoptosis by up-regulating TRAIL/Apo2L death receptor 5 expression in LNCaP cells. LNCaP cells were exposed to ALLN for $3 \mathrm{~h}$ and treated with recombinant TRAIL protein. ALLN alone induced a $20 \%$ cell death after a 3 -h treatment; however, pretreatment with ALLN induced death to more than $80 \%$ of cells after $3 \mathrm{~h}$ of TRAIL treatment. ALLN also enhanced the cell death of TRAIL-sensitive/resistant prostate cancer and other cancer cell lines. Western blotting results showed that the combination of ALLN and TRAIL increased the levels of activated caspase-8, -3 and DR-5 in LNCaP cells. Furthermore, we observed an increase in DR-5 expression following $3 \mathrm{~h}$ of treatment of ALLN alone. Taken together, our findings indicate that ALLN enhances TRAIL-induced apoptosis in LNCaP cells by up-regulating DR-5 expression. Thus, our results suggest that the combination of ALLN and TRAIL is a novel therapeutic strategy in TRAIL-resistant tumors.
\end{abstract}

\section{Introduction}

Prostate cancer is the most frequently diagnosed male cancer and the second leading cause of cancer mortality in men (1). Chemotherapy and androgen withdrawal are used for slowing down the spread of the disease. However, no effective treatments are currently available for advanced prostate cancer. Therefore, a better understanding of cancer cell survival

Correspondence to: Dr Sang-Youel Park, College of Veterinary Medicine, Chonbuk National University, Jeonju, Jeonbuk 561-756, Republic of Korea

E-mail: sypark@chonbuk.ac.kr

Key words: proteasome inhibitor, ALLN, tumor necrosis factorrelated apoptosis inducing ligand, apoptosis, death receptor 5 mechanisms and more efficient therapy methods are required for the treatment of prostate cancer.

Tumor necrosis factor-related apoptosis inducing ligand (TRAIL), a member of the TNF superfamily, selectively induces apoptosis in cancer cells with no toxicity against normal tissues $(2,3)$. TRAIL induces apoptosis by first binding to its membrane death receptors, including death receptor 4 (DR-4) and DR-5, causing the formation of a death-inducing signaling complex (DISC), which includes the receptors, the adaptor protein FADD and caspase-8. However, some tumor cells are resistant to TRAIL-mediated cytotoxicity $(4,5)$. A decreased expression of DR-4 and DR-5 or an increased expression of antiapoptotic proteins in cancer cells is involved in TRAIL-resistance. Prostate cancer often expresses TRAIL receptors on the cell surface. However. these receptors are often not capable of triggering TRAIL-induced apoptosis due to increased pro-survival signaling (6). Research is focused on identifying combination therapies with agents that reduce TRAIL resistance without harming normal tissues.

Ubiquitin-proteasome mediated protein degradation pathway plays an important role in regulating cell proliferation and cell death (7). This process suggests that degradation of specific cell proteins is involved in determining cell proliferation or cell death. Inhibition of the ubiquitin-proteasome system by proteasome inhibitors blocks the process of programmed cell death in thymocytes and neurons, but induces apoptosis in various human cancer cell lines (8-10). Moreover, to sensitize TRAIL-resistant cancer cells, proteasome inhibitors are combined with TRAIL in a variety of different cancer models. For example, the proteasome inhibitors PS-341 and MG-341 were shown to help overcome TRAIL-resistant cancer cells to the proapoptotic effect of TRAIL (11-13).

In the present study, we investigated the enhancing effect of N-acetyl-leucinyl-leucinyl-norleucinal (ALLN), known as calpain inhibitor 1 , as a proteasome inhibitor on TRAILresistant prostate cancer cells.

\section{Materials and methods}

Cell culture and reagents. LNCaP, HPC-36, PC-3 and DU145 (prostate cancer), MCF7 (breast cancer), A549 (lung cancer), HCT116 (colon cancer) and SNU638 (gastric cancer) cell lines were obtained from the American Type Culture Collection (ATCC) and maintained in RPMI-1640 and DMEM (Gibco 

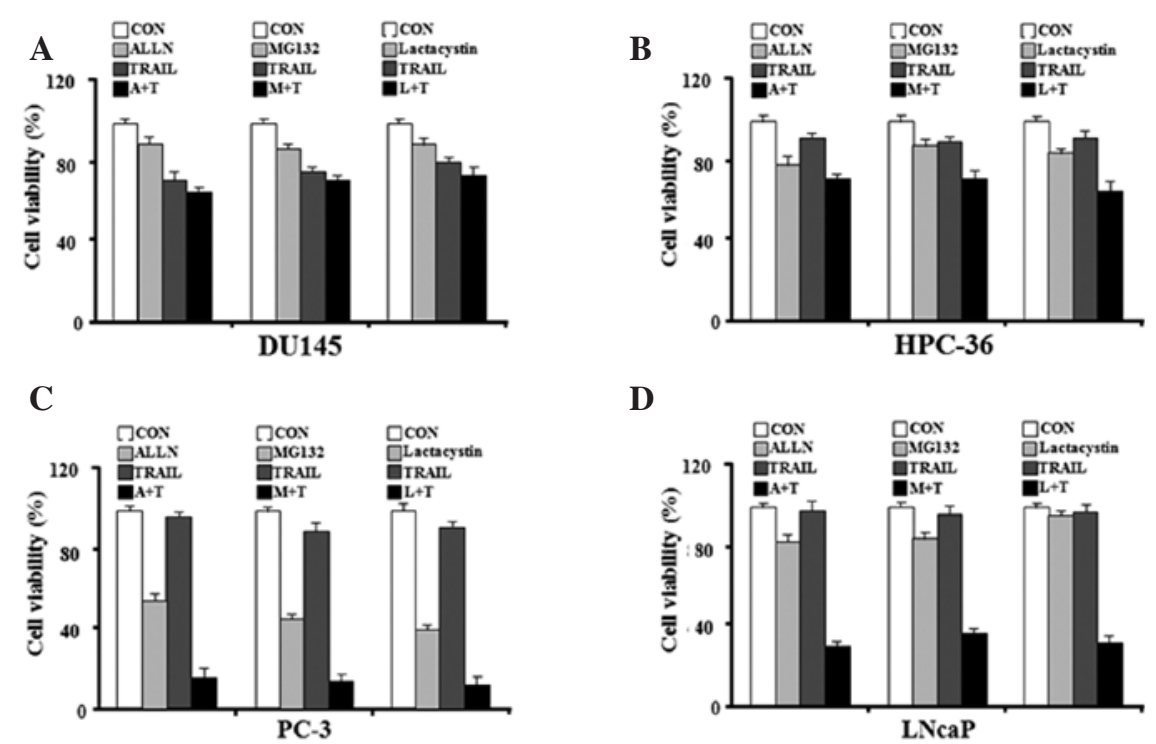

D

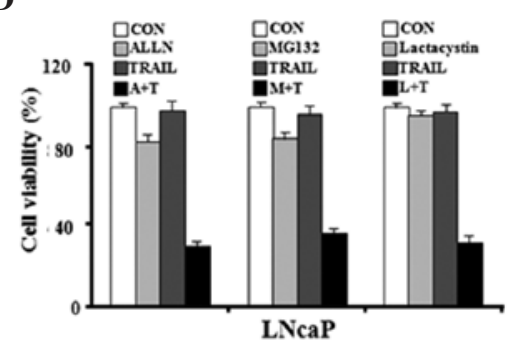

Figure 1. Cell viability after treatment with TRAIL and/or proteasome inhibitors in prostate cancer cells. Prostate cancer cells were plated in 96-well plates and incubated overnight. (A) DU145, (B) HPC-36, (C) PC-3 and (D) LNCaP cells were exposed to ALLN (10 $\mu \mathrm{M})$, MG-132 (10 $\mu \mathrm{M})$ and lactacystin (5 $\mu \mathrm{M})$ for $3 \mathrm{~h}$ and then treated with recombinant TRAIL $(100 \mathrm{ng} / \mathrm{ml})$ protein for an additional $3 \mathrm{~h}$. Cell viability was determined by crystal violet staining. Viability of the control cells was set at $100 \%$; viability relative to the control is shown. The experiments were performed in triplicate, at least twice. Bar, standard deviation.

BRL, Carlsbad, CA, USA) culture medium supplemented with $10 \%(\mathrm{v} / \mathrm{v})$ fetal bovine serum and antibiotics $(100 \mu \mathrm{g} /$ $\mathrm{ml}$ gentamycin and $100 \mu \mathrm{g} / \mathrm{ml}$ penicillin-streptomycin). Proteasome inhibitors such as lactacystin (AG Scientific, Inc., San Diego, CA, USA), MG-132 and ALLN (Sigma, St.Louis, MO, USA) was dissolved in dimethylsulphoxide (DMSO).

Cell viability. Whole cells were plated at $1.0 \times 10^{4}$ cells in a 12-well plate, and incubated at $37^{\circ} \mathrm{C}$ for $24 \mathrm{~h}$. The cells were pretreated with ALLN, lactacystin and MG-132 (0-40 $\mu \mathrm{M})$ for 0-3 $\mathrm{h}$ and further incubated with recombinant TRAIL protein (14) for an additional $3 \mathrm{~h}$. Cell morphology images were captured under a microscope (inverted Microscope, NIKON, Japan), and cell viability was determined by the crystal violet staining method as previously described (15). Briefly, cells were stained for $10 \mathrm{~min}$ at room temperature with a staining solution ( $0.5 \%$ crystal violet in 30\% ethanol and 3\% formaldehyde), washed 4 times with water, and dried. Cells were then lysed with $1 \%$ SDS solution, and measured at $550 \mathrm{~nm}$. Cell viability was calculated from the relative dye intensity and compared to the controls.

Western blot analysis. To prepare whole cell lysates, cells were harvested, resuspended in lysis buffer ( $25 \mathrm{mM}$ HEPES (pH 7.4), $100 \mathrm{mM} \mathrm{NaCl}, 1 \mathrm{mM}$ EDTA, $5 \mathrm{mM} \mathrm{MgCl} 2,0.1 \mathrm{mM}$ DTT and protease inhibitor mixture) and sonicated. Proteins were separated on an 8-15\% SDS gel and subjected to Western blot analyses as previously described $(16,17)$. The proteins $(40 \mu \mathrm{g} / \mathrm{ml})$ were separated on a $12-15 \%$ SDS gel and transferred to a nitrocellulose membrane. After incubation for 1-24 $\mathrm{h}$ with a 1:1000 primary antibody dilution buffer (1\% milk with PBS-Tween), the membranes were developed by enhanced chemiluminescence using a secondary antibody dilution. The antibodies used for the Western blot analyses were DR-4 (AAP420), DR-5 (AAP-430), caspase-8 (AAP-118) and caspase-3 (AAP-103),obtained from Stressgen (Victoria, Canada).
Statistical analysis. Data are expressed as the mean \pm standard $\mathrm{SD}$. The data were compared using the Student's t-test and the ANOVA Duncan test with the SAS statistical package. The results were considered significant at values of ${ }^{*} \mathrm{P}<0.05$ or ${ }^{* * *} \mathrm{P}<0.01$.

\section{Results}

Proteasome inhibition sensitizes various TRAIL-sensitive/ resistant cancer cells. We investigated the interactions between TRAIL and proteasome inhibitors on various prostate cancer cell lines. Whole cells were exposed to ALLN $(10 \mu \mathrm{M})$, MG-132 $(10 \mu \mathrm{M})$ and lactacystin $(5 \mu \mathrm{M})$ for $3 \mathrm{~h}$ and then treated with recombinant TRAIL $(100 \mathrm{ng} / \mathrm{ml})$ protein for an additional $3 \mathrm{~h}$. TRAIL treatment induced approximately $25 \%$ cell death in DU145 (Fig. 1A), but TRAIL alone had no cytotoxic effect on HPC-36, PC-3 and LNCaP cells (Fig. 1B-D). However, when a combination therapy of proteasome inhibitors and TRAIL was used, whole prostate cancer cells significantly decreased cell viability as compared to TRAIL alone.

We then examined the combination effect of TRAIL with proteasome inhibitor, particularly ALLN, in various cancer cells. These cells were exposed to ALLN for $3 \mathrm{~h}$ and treated with recombinant TRAIL $(100 \mathrm{ng} / \mathrm{ml})$ protein for an additional $3 \mathrm{~h}$. ALLN alone induced an approximately 10-30\% apoptosis in prostate (Fig. 2A) as well as other cancer cells (Fig. 2B). However, the combined treatment of ALLN and TRAIL increased cell death in whole cancer cells, although TRAIL alone exhibited a reduced effect on cancer cell death. Notably, the combined treatment induced almost 70-80\% cell death in PC-3 and $\mathrm{LNCaP}$ cells. The cell morphology images also confirmed this enhanced cell death activity by the combined treatment of ALLN and TRAIL in various cancer cells (Fig. 2C).

Proteasome inhibitor ALLN sensitizes TRAIL-resistant $L N C a P$ prostate cancer cells. To examine the functional role 

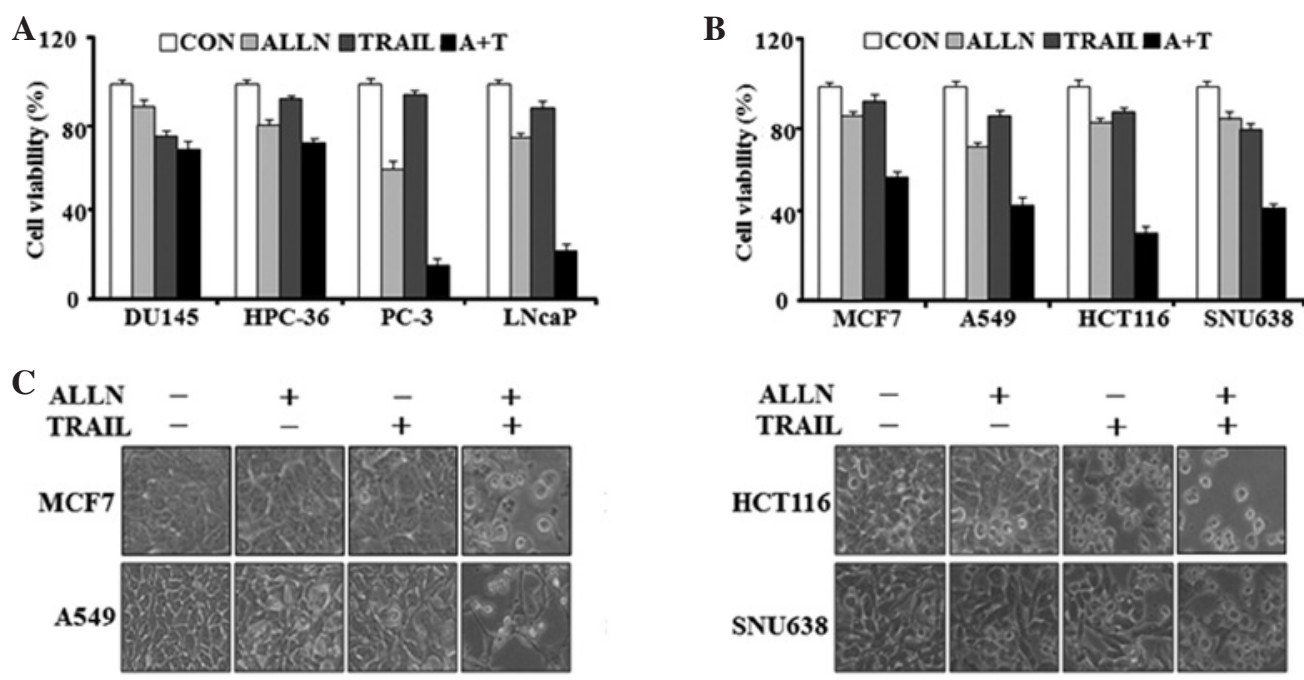

Figure 2. Cell viability after treatment with TRAIL and/or ALLN in various cancer cells. Prostate and other cancer cells were plated in the 96-well plates and incubated overnight. (A) Prostate (B) other cancer cells were exposed to ALLN $(10 \mu \mathrm{M})$ for $3 \mathrm{~h}$ and then treated with recombinant TRAIL (100 ng/ml) protein for an additional $3 \mathrm{~h}$. Cell viability was determined by crystal violet staining. Viability of control cells was set at 100\%; viability relative to the control is shown. The experiments were performed in triplicate, at least twice. Bar, standard deviation. (C) Cell morphology images under the conditions described in (B) were captured.
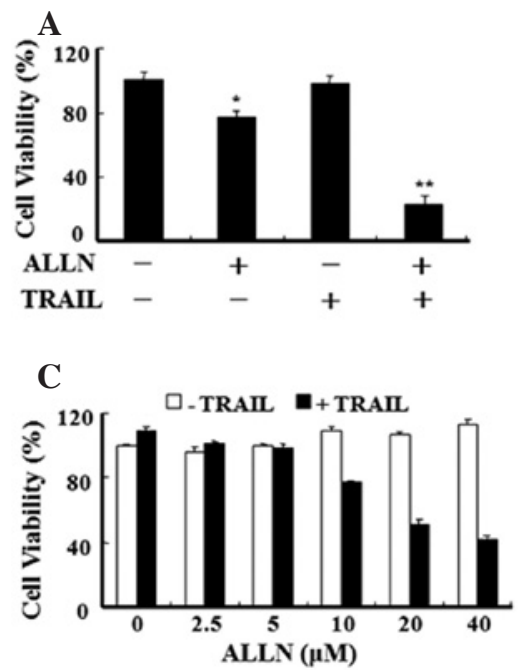

B

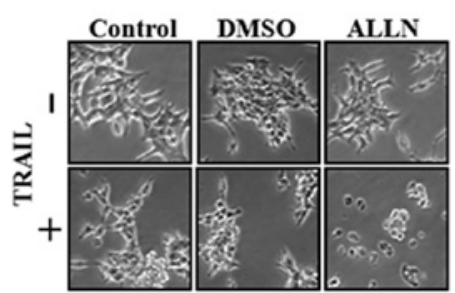

D

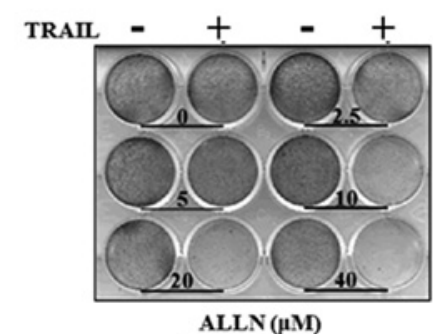

Figure 3. Effect of ALLN on TRAIL-resistant LNCaP prostate cancer cells. (A) LNCaP cells cultured in 12-well plates were pre-treated with ALLN (10 $\mu \mathrm{M})$ for $3 \mathrm{~h}$ and then further co-incubated with recombinant TRAIL protein $(100 \mathrm{ng} / \mathrm{ml})$ for an additional $3 \mathrm{~h}$. Cell viability was determined by crystal violet staining. The experiments were performed in triplicate, at least twice. Bar, standard error. ${ }^{* *} \mathrm{P}<0.01,{ }^{*} \mathrm{P}<0.05$ vs. normal samples were calculated using the Student's t-test. (B) Cell morphology under the conditions described in (A) was captured. (C) LNCaP cells cultured in 12-well plates were pre-treated with ALLN $(0-40 \mu \mathrm{M})$ for $3 \mathrm{~h}$, and then further co-incubated with or without recombinant TRAIL protein $(100 \mathrm{ng} / \mathrm{ml})$ for an additional $3 \mathrm{~h}$. Cell viability was determined as described (A). (D) A set of 12-well plates showing cell viability of the conditions described in (C) is shown.

of ALLN in apoptotic cell death, LNCaP cells, which are resistant to apoptotic induction by TRAIL alone, were exposed to ALLN $(10 \mu \mathrm{M})$ for $3 \mathrm{~h}$ and then treated with recombinant TRAIL protein for an additional $3 \mathrm{~h}$. ALLN alone induced approximately $20 \%$ apoptosis in the LNCaP cells, whereas pretreatment with ALLN increased TRAIL-induced apoptosis to $80 \%$, although TRAIL alone exhibited a reduced effect on tumor cell viability (Fig. 3A). Examination of cell morphology also verified this enhanced activity of ALLN on TRAILinduced apoptosis (Fig. 3B). Furthermore, LNCaP cells were pretreated with ALLN in a dose-dependent manner for $3 \mathrm{~h}$ and then coincubated with and without TRAIL protein for an additional $3 \mathrm{~h}$. As shown in Fig. 3C, ALLN enhanced the TRAIL effect in a concentration-dependent manner. Moreover, a marked increase was found in TRAIL-induced apoptosis at a high concentration (20 and $40 \mu \mathrm{M})$. Following treatment, the 12-well plate was stained with a crystal violet staining method in which purple staining was evident when cells were viable. After staining, the 12-well plates were scanned. The scanning results clearly indicated that the cell viability for ALLN and TRAIL was markedly depressed (Fig. 3D).

Proteasome inhibitor ALLN increases DR-5 proteins in LNCaP prostate cancer cells. To address the mechanism by which ALLN enhances TRAIL-induced apoptosis, we first examined caspase- 8 and -3 activation after treating the cells with ALLN and/or TRAIL (Fig. 4 A and B). Under normal growth conditions and treatment of ALLN cells, Western blotting did not 
A

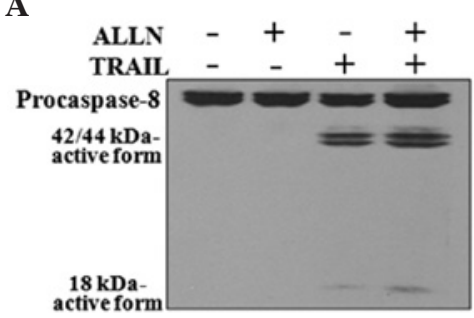

C

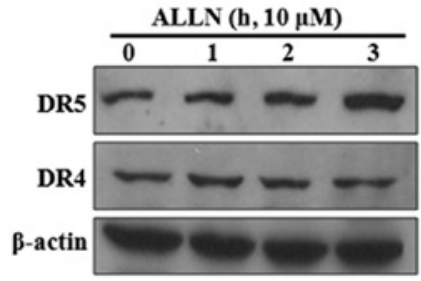

B

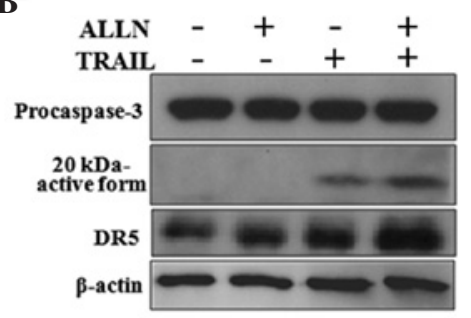

D

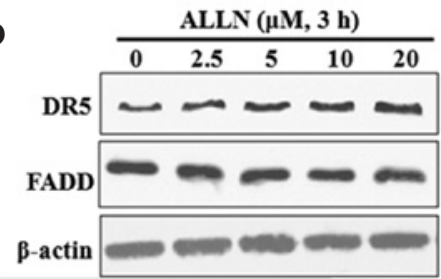

Figure 4. ALLN enhanced TRAIL-induced apoptosis by DR-5 upregulation and caspase-8 and -3 activation. (A and B) LNCaP cells were pre-treated with ALLN for $3 \mathrm{~h}$, and then further co-incubated with or without recombinant TRAIL protein $(100 \mathrm{ng} / \mathrm{ml})$ for an additional $1 \mathrm{~h}$. Whole cell lysates were prepared and protein samples were separated on SDS-PAGE, analyzed for apoptotic proteins such as caspase- 8 and -3 by Western blotting analysis. (C) LNCaP cells were pre-treated with ALLN $(10 \mu \mathrm{M})$ for $0-3 \mathrm{~h}$ and the prepared samples were separated on SDS-PAGE and analyzed for DR-4 and DR-5 protein expression. (D) ALLN was treated with $0-20 \mu \mathrm{M}$ and Western blotting analysis was performed to analyze the FADD and DR-5 expression. $\beta$-actin was used as the control for protein loading.

efficiently detect an active form of caspase-8 and -3 . However, treatment with TRAIL protein alone and the combined treatment of ALLN and TRAIL protein induced caspase-8 and -3 activation. More importantly, cells co-treated with ALLN and TRAIL protein showed higher activation levels of caspase- 8 and -3 than cells treated with TRAIL alone. Although the cells treated with TRAIL alone exhibited an activation of caspase- 8 and-3, the cells were resistant to TRAIL-induced apoptosis, indicating that the caspase- 8 and -3 activation by TRAIL alone was not sufficient to induce apoptosis. However, pretreatment with ALLN enhanced the activation of the two proteins, thereby enhancing TRAIL-induced apoptosis. As previously described, TRAIL-induced apoptotic signaling occurs via DR-4/DR-5 receptors, which ultimately leads to apoptosis through caspase activation. Since ALLN enhanced the TRAIL effect in LNCaP cells by an increased activation of caspase- 8 and -3 , it may be combined with DR-4/DR-5 up-regulation by ALLN. Therefore, to elucidate the effect of ALLN on DR-4/ DR-5 up-regulation, we further investigated the DR-4/DR-5 protein levels by Western blotting. We first exposed LNCaP cells to ALLN for different time periods (Fig. 4C). ALLN was found to enhance DR-5 protein expression after $3 \mathrm{~h}$ of treatment. However, no significant increase was observed in DR-4 protein expression even after the $3 \mathrm{~h}$ treatment. Furthermore, the cells were treated with different ALLN concentrations and the DR-5 protein expression was examined. Fig. 4D shows that ALLN also increased DR-5 expression in a concentrationdependent manner. By up-regulating DR-5 protein expression, we showed that ALLN markedly enhanced anti-tumorigenic effects in TRAIL-resistant prostate cancer cells.

\section{Discussion}

Proteasome inhibitors are attractive cancer therapeutic agents as they are capable of regulating apoptotis-related proteins. Various proteasome inhibitors such as Bortezomib, MG132, PS-341 and ALLN have been reported to induce apoptotic cell death in various cell lines $(10,18-21)$. When used in combination with TRAIL, some proteasome inhibitors enhanced TRAILinduced cell death $(9,11,21-25)$. For example, by up-regulating DR-5, MG132 cooperates with TRAIL to overcome the Bax deficiency-induced resistance to TRAIL in HCT-116 cells (26). Studies have also demonstrated that ALLN regulates apoptotic cell death with marked increases in a large number of cellular proteins, including cyclin-dependent protein kinase inhibitor p21WAF1 $(8,27)$. However, little is known about the effect of ALLN on TRAIL-induced apoptosis. In the present study, we investigated the interactions between TRAIL and proteasome inhibitors on various prostate cancer cell lines. Our results showed that when proteasome inhibitors and TRAIL were used in combination, whole prostate cancer cells significantly decreased cell viability compared to TRAIL alone. In particular, the combination of TRAIL with the proteasome inhibitor, ALLN, increased cell death in various cancer cell lines although a decreased effect on cancer cell death was observed with tRAIL alone. These data suggest that the proteasome inhibitors ALLN, sensitizes on TRAIL-sensitive/ resistant prostate cancer and other cancer cells.

Apoptosis induced by TRAIL is characterized by binding with its death receptors, such as DR-4 and DR-5, and forming of the death-inducing signaling complex (DISC) $(28,29)$. DISC includes receptors, the adaptor FADD protein and the caspase-8. Therefore, caspase- 8 and -3 activation was first examined after treating the cells with ALLN and/or TRAIL in order to address the mechanism by which ALLN enhances TRAIL-induced apoptosis. The cells treated with both ALLN and TRAIL showed an increase in the activation of the caspase- 8 and -3 protein compared to cells treated with TRAIL alone. These results suggest that although the cells treated with TRAIL alone showed caspase- 8 and -3 activation, pretreatment with ALLN enhanced the activation of the two proteins, which, in turn, enhanced the TRAIL-induced apoptosis. In addition, these results indicate that ALLN can be combined with DR4/DR5 up-regulation in enhancing the 
mechanism of ALLN on TRAIL-induced LNCaP cell death as a result of the increased caspase- 8 and -3 activation.

Other proteasome inhibitors such as MG-132, Bortezomib and PS-341 have been reported to up-regulate DR-5 protein expression thereby enhancing TRAIL-induced apoptosis in different cell lines $(7,11,30,31)$. MG-132 induced DR-5 protein expression in prostate cancer cells in a dose-dependent manner and thus enhanced TRAIL-induced apoptosis (30). It has been shown that MG-132 elevated the levels of TRAIL receptors such as DR-4 and DR-5 in HeLa cells (32). Furthermore, Bortezomib and MG-132, in combination, induced only DR-5, but not DR-4, expression in human colon cancer cell lines (31). Similarly, we present data for ALLN in LNCaP cells whereby ALLN alone induced DR-5 expression, without any effect on DR-4. We also showed that DR-5 expression was induced by the combined treatment of ALLN and TRAIL. Our results showed that ALLN enhanced TRAIL-induced apoptosis in LNCaP cells by up-regulating DR-5 expression and subsequently by caspase- 8 and -3 activation.

In conclusion, the proteasome inhibitor ALLN enhanced the TRAIL effect in TRAIL-resistant LNCaP cells by up-regulating TRAIL receptor DR-5. Therefore, ALLN may be used in synergy with TRAIL and can be proved a successful chemotherapeutic combination for tumor cells, particularly those resistant to TRAIL-induced apoptosis.

\section{Acknowledgements}

This work was supported by Regional Research Universities Program and the Center for Healthcare Technology Development and National Research Foundation of Korea Grant funded by the Korean Government (2011-0003717).

\section{References}

1. Jemal A, Siegel R, Ward E, Murray T, Xu J and Thun MJ: Cancer statistics. CA Cancer J Clin 57: 43-66, 2007.

2. Griffith TS, Anderson RD, Davidson BL, Williams RD and Ratliff TL: Adenoviral-mediated transfer of the TNF-related apoptosis-inducing ligand/Apo-2 ligand gene induces tumor cell apoptosis. J. Immunol 165: 2886-2894, 2000.

3. Walczak H, Miller RE, Ariail K, et al: Tumoricidal activity of tumor necrosis factor-related apoptosis- inducing ligand in vivo. Nat Med 5: 157-163, 1999.

4. Malhi $\mathrm{H}$ and Gores GJ: TRAIL resistance results in cancer progression: a TRAIL to perdition? Oncogene 25: 7333-7335, 2006.

5. Zhang X, Jin TG, Yang H, DeWolf WC, Khosravi-Far R and Olumi AF: Persistent c-FLIP(L) expression is necessary and sufficient to maintain resistance to tumor necrosis factor-related apoptosis-inducing ligand-mediated apoptosis in prostate cancer. Cancer Res 64: 7086-7091, 2004.

6. Bucur O, Ray S, Bucur MC and Almasan A: APO2 ligand/tumor necrosis factor-related apoptosis-inducing ligand in prostate cancer therapy. Front Biosci 11: 1549-1568, 2006.

7. Ishizawa J, Yoshida S, Oya M, et al: Inhibition of the ubiquitin-proteasome pathway activates stress kinases and induces apoptosis in renal cancer cells. Int J Oncol 25: 697-702, 2004

8. An WG, Hwang SG, Trepel JB and Blagosklonny MV: Protease inhibitor-induced apoptosis: accumulation of wt p53, p21WAF1/ CIP1, and induction of apoptosis are independent markers of proteasome inhibition. Leukemia 14: 1276-1283, 2000.

9. Inoue T, Shiraki K, Fuke H, et al: Proteasome inhibition sensitizes hepatocellular carcinoma cells to TRAIL by suppressing caspase inhibitors and AKT pathway. Anticancer Drugs 17: 261-268, 2006

10. Nawrocki ST, Carew JS, Pino MS, et al: Bortezomib sensitizes pancreatic cancer cells to endoplasmic reticulum stress-mediated apoptosis. Cancer Res 65: 11658-11666, 2005.
11. Johnson TR, Stone K, Nikrad M, et al: The proteasome inhibitor PS-341 overcomes TRAIL resistance in Bax and caspase 9negative or Bcl-xL overexpressing cells. Oncogene 22: 4953-4963, 2003.

12. Sayers TJ, Brooks AD, Koh CY, et al: The proteasome inhibitor PS-341 sensitizes neoplastic cells to TRAIL-mediated apoptosis by reducing levels of c-FLIP. Blood 102: 303-310, 2003.

13. Sohn D, Totzke G, Essmann F, Schulze-Osthoff K, Levkau B and Janicke RU: The proteasome is required for rapid initiation of death receptor-induced apoptosis. Mol Cell Biol 26: 1967-1978, 2006.

14. Seol DW and Billiar TR: Cysteine 230 modulates tumor necrosis factor-related apoptosis-inducing ligand activity. Cancer Res 60: 3152-3154, 2000

15. Seol DW and Billiar TR: A caspase-9 variant missing the catalytic site is an endogenous inhibitor of apoptosis. J Biol Chem 274: 2072-2076, 1999.

16. Park SY,Billiar TR and Seol DW: Hypoxia inhibition of apoptosis induced by tumor necrosis factor-related apoptosis-inducing ligand (TRAIL). Biochem Biophys Res Commun 291: 150-153, 2002.

17. Seol DW, Li J, Seol MH, Park SY, Talanian RV and Billiar TR: Signaling events triggered by tumor necrosis factor-related apoptosis- inducing ligand (TRAIL): caspase-8 is required for TRAIL-induced apoptosis. Cancer Res 61: 1138-1143, 2001.

18. LuM,DouQP,Kitson RP,Smith DM andGoldfarbRH:Differential effects of proteasome inhibitors on cell cycle and apoptotic path-ways in human YT and Jurkat cells. J Cell Biochem 97: 122-134, 2006.

19. Yin D, Zhou H, Kumagai T, et al: Proteasome inhibitor PS-341 causes cell growth arrest and apoptosis in human glioblastoma multiforme (GBM). Oncogene 24: 344-354, 2005.

20. Ling YH, Liebes L, Ng B, et al: PS-341, a novel proteasome inhibitor, induces Bcl-2 phosphorylation and cleavage in association with G2-M phase arrest and apoptosis. Mol Cancer Ther 1: 841-849, 2002.

21. Wente MN, Eibl G, Reber HA, Friess H, Buchler MW and Hines OJ: The proteasome inhibitor MG132 induces apoptosis in human pancreatic cancer cells. Oncol Rep 14: 1635-1638, 2005.

22. Brooks AD, Ramirez T, Toh U, et al: The proteasome inhibitor bortezomib (Velcade) sensitizes some human tumor cells to apo2L/TRAIL-mediated apoptosis. Ann N Y. Acad Sci 1059: $160-167,2005$

23. Ganten TM, Koschny R, Haas TL, et al: Proteasome inhibition sensitizes hepatocellular carcinoma cells, but not human hepatocytes, to TRAIL. Hepatology 42: 588-597, 2005.

24. Lashinger LM, Zhu K, Williams SA, Shrader M, Dinney CP and McConkey DJ: Bortezomib abolishes tumor necrosis factorrelated apoptosis-inducing ligand resistance via a p21-dependent mechanism in human bladder and prostate cancer cells. Cancer Res 65: 4902-4908, 2005.

25. Mlynarczuk I, Hoser G, Grzela T, et al: Augmented pro-apoptotic effects of TRAIL and proteasome inhibitor in human promonocytic leukemic U937 cells. Anticancer Res 21: 1237-1240, 2001.

26. He Q, Huang Y and Sheikh MS: Proteasome inhibitor MG132 upregulates death receptor 5 and cooperates with Apo2L/ TRAIL to induce apoptosis in Bax-proficient and-deficient cells. Oncogene 23: 2554-2558, 2004.

27. Kim OH, Lim JH, Woo KJ, et al: Influence of p53 and p21Waf1 expression on $\mathrm{G} 2 / \mathrm{M}$ phase arrest of colorectal carcinoma HCT116 cells to proteasome inhibitors. Int J Oncol 24: 935-941, 2004.

28. Pitti RM, Marsters SA, Ruppert S, Donahue CJ, Moore A and Ashkenazi A: Induction of apoptosis by Apo-2 ligand, a new member of the tumor necrosis factor cytokine family. J Biol Chem. 271: 12687-12690, 1996.

29. Wiley SR, Schooley K, Smolak PJ, et al: Identification and characterization of a new member of the TNF family that induces apoptosis. Immunity 3: 673-682, 1995.

30. Yoshida T, Shiraishi T, Nakata S, et al: Proteasome inhibitor MG132 induces death receptor 5 through CCAAT/enhancerbinding protein homologous protein. Cancer Res 65: 5662-5667, 2005.

31. Zhu H, Guo W, Zhang L, et al: Proteasome inhibitors-mediated TRAIL resensitization and Bik accumulation. Cancer Biol Ther 4: 781-786, 2005.

32. Hougardy BM, Maduro JH, van der Zee AG, et al: Proteasome inhibitor MG132 sensitizes HPV-positive human cervical cancer cells to rhTRAIL-induced apoptosis. Int J Cancer 118: 1892-1900, 2006. 